\title{
Classification of certain 6-manifolds
}

\author{
By P. E. JUPP \\ University of Geneva†
}

(Received 19 November 1971)

0. Introduction. In (13) Wall classified up to diffeomorphism, PL-homeomorphism, topological homeomorphism, and homotopy equivalence all closed, oriented, 1connected smooth (or PL) 6-manifolds with torsion-free homology and vanishing second Stiefel-Whitney class. This paper extends these classifications to all closed, oriented, 1-connected topological 6-manifolds with torsion-free homology. The method differs from that of (13) but uses two special cases of Wall's classification.

We shall work in the categories of smooth, PL, and topological manifolds and maps, and 'manifold', 'homeomorphism', etc., are to be interpreted consistently in any one of these categories.

I am grateful to the Royal Society for a Fellowship in the European Programme, during the tenure of which the work described in this paper was carried out.

1. Invariants of the manifolds. Let $M$ be a closed, oriented, 1-connected 6-manifold with torsion-free homology. The cohomology of $M$ is determined by: $H=H^{2}(M)$, a finitely generated free abelian group; $r=\operatorname{rank} H^{3}(M) / 2$, a non-negative integer; $\mu: H \otimes H \otimes H \rightarrow \mathbb{Z}$, a symmetric homomorphism given by the cup-product evaluated on the fundamental class. There are also the following characteristic classes:

$$
w \in H^{2}(M ; \mathbb{Z})=H \otimes \mathbb{Z}_{2},
$$

the second Stiefel-Whitney class; $p \in H^{4}(M ; \mathbb{Z})=\operatorname{Hom}_{\mathbb{Z}}(H, \mathbb{Z})$, the first Pontrjagin class; $\Delta \in H^{4}\left(M ; \mathbb{Z}_{2}\right)=\operatorname{Hom}_{\mathbb{Z}}\left(H, \mathbb{Z}_{2}\right)$, the triangulation class (the obstruction to lifting the stable tangent bundle of $M$ to a PL bundle (8)). (That the first Pontrjagin class of a topological bundle is an integral class can be seen as follows. The generator of $\mathbb{Z}_{2}=\pi_{3}(T O P / P L) \subset \pi_{4}(B T O P)((10), 5 \cdot 5)$ can be represented by a map $S^{4} \cup_{2} e^{5} \rightarrow B T O P$. As $B T O P$ has the homotopy type of a loop space(1), we can combine this map with the inclusion of $B O$ in $B T O P$ to get a map $B O \times\left(S^{4} \cup_{2} e^{5}\right) \rightarrow B T O P$. This map is 5connected. It follows that the inclusion of $B O$ in $B T O P$ induces an isomorphism

$$
\left.H^{4}(B T O P ; \mathbb{Z}) \rightarrow H^{4}(B O ; \mathbb{Z}) .\right)
$$

We obtain relations between the invariants by applying the $\hat{A}$-integrality theorem ((5), 26.1.1). (This was suggested by E.G. Rees.) As $H^{2}\left(M ; \mathbb{Z}_{2}\right)=H \otimes \mathbb{Z}_{2}$, there is $W \in H$ which reduces mod 2 to $w$. For $x \in H$ there is a complex line bundle $\eta$ over $M$

$\dagger$ Present address: Pembroke College, Cambridge. 
with first Chern class $x((6)$, thm. 3.4, p. 234). Also $c h(\eta)=\exp (x)((5), 10 \cdot 1)$. Suppose $M$ is smooth, then by the $\hat{A}$-integrality theorem

$$
\begin{gathered}
\langle\exp (W / 2) \cdot \exp (x) \cdot(1-p / 24),[M]\rangle \in \mathbb{Z}, \\
\mu(W+2 x, W+2 x, W+2 x) \equiv p(W+2 x) \quad(\bmod 48) .
\end{gathered}
$$

To"get a similar relation for topological manifolds we argue as follows. The obstruction to lifting the stable normal spherical fibration of $M$ to a vector bundle is an element of $H^{3}\left(M ; \pi_{2}(G / O)\right)$ which has image $O \in H^{3}\left(M ; \pi_{2}(G / T O P)\right)$ under the obvious map of coefficients. As $\pi_{2}(G / O) \rightarrow \pi_{2}(G / T O P)$ is an isomorphism, the above obstruction vanishes. We may then do surgery as in the proof of Theorem 8 of (13) to get a smooth manifold $M^{\prime}$ homotopy equivalent to $M$. The stable tangent bundles of $M$ and $M^{\prime}$ are classified by elements of $[M ; B T O P]$ which have the same image in $[M ; B G]$. As $\pi_{2}(B T O P)=\pi_{2}(B G)$, the bundles restricted to a 2-skeleton are isomorphic, and there is a difference element in $H^{4}\left(M ; \pi_{4}(B T O P)\right)$ with image $O \in H^{4}\left(M ; \pi_{4}(B G)\right)$. Now $\mathbb{Z}=\pi_{4}(B O) \rightarrow \pi_{4}(B G)=\mathbb{Z}_{24}$ is reduction mod 24 , it follows from $((10), 5 \cdot 5)$ that

$$
\mathbb{Z}_{2}=\pi_{3}(T O P \mid P L) \rightarrow \pi_{3}(G)=\mathbb{Z}_{24}
$$

is non-trivial, and the generator of $\pi_{4}(B O)$ defines a bundle with Pontrjagin class 2 (12). Thus the stable tangent bundles of $M$ and $M^{\prime}$ have the same value of $p+24 T \bmod 48$, where $T \in \operatorname{Hom}_{\mathbb{Z}}(H, \mathbb{Z})$ reduces $\bmod 2$ to $\Delta$. As $\mu$ and $w$ are homotopy invariants, we have

$$
\mu(2 x+W, 2 x+W, 2 x+W) \equiv(p+24 T)(2 x+W) \quad(\bmod 48) .
$$

It will follow from Theorem 1 that there are essentially no other relations.

2. The classification. As a first step towards a normal form for our manifolds we can split off copies of $S^{3} \times S^{3}$. The smooth and $P L$ cases of the following are Theorem 1 of (13) - the topological case is proved analogously.

Theorem 0. Let $M$ be a closed, oriented, 1-connected 6-manifold. Then

where $H_{3}\left(M_{1}\right)$ is finite.

$$
M=M_{1} \#\left(\#_{1}^{+} S^{3} \times S^{3}\right) \text {, }
$$

Thus we need consider only manifolds $M$ with $H_{3}(M)=0$, and this we now do.

Definition. $A$ based manifold of rank $s$ is a pair $(M, \mathbf{e})$ where $M$ is a closed, oriented, 1-connected 6-manifold with $H_{2}(M)=\oplus_{1}^{8} \mathbb{Z}, H_{3}(M)=0$, and $\mathbf{e}=\left\{e_{i}: 1 \leqslant i \leqslant s\right\}$ is a base of $H_{2}(M)$ such that, for $i>1, w\left(e_{i}\right)=.0 \in \mathbb{Z}_{2}$, where $w \in H^{2}\left(M ; \mathbb{Z}_{2}\right)$ is the second StiefelWhitney class. $A$ based homeomorphism of based manifolds $f:(M, \mathbf{e}) \rightarrow\left(M^{\prime}, \mathbf{e}^{\prime}\right)$ is an orientation-preserving homeomorphism $f: M \rightarrow M^{\prime}$ such that $f_{*}\left(e_{i}\right)=e_{1}^{\prime}$.

Let $S^{2} \widetilde{\times} D^{4}$ denote the total space of the non-trivial $D^{4}$-bundle over $S^{2}$, and put $B_{s}^{+}=+{ }_{1}^{s}\left(S^{2} \times D^{4}\right), B_{s}^{-}=\left(S^{2} \widetilde{\times} D^{4}\right)+\left(+_{1}^{s-1}\left(S^{2} \times D^{4}\right)\right)$, where + denotes boundary connected sum. Choose an orientation of $B_{s}^{ \pm}$and choose $\bar{e}_{i} \in H_{2}\left(\partial B_{s}^{ \pm}\right), 1 \leqslant i \leqslant s$, so that $\bar{e}_{i}$ is the image of a fundamental class of the $i$ th copy of $S^{2}$.

Proposition 1. There is a bijection from the set of concordance classes of orientationpreserving homeomorphisms of $\partial B_{s}^{+}\left(\partial B_{s}^{-}\right)$which induce the identity on $H_{2}\left(\partial B_{s}^{+}\right)\left(H_{2}\left(\partial B_{s}^{-}\right)\right)$ 
to the set $\mathbf{M}_{s}^{+}\left(\mathbf{M}_{s}^{-}\right)$of based homeomorphism classes of based manifolds of rank $s$ with $w=0(w \neq 0)$.

Proof. If $f$ is an orientation-preserving homeomorphism of the type considered, then $B_{s}^{ \pm} \cup_{f} B_{s}^{ \pm}$with orientation given by the chosen one on the first copy of $B_{s}^{ \pm}$and with base of $H_{2}\left(B_{s}^{ \pm} \cup_{f} B_{s}^{ \pm}\right)$given by the image of $\left\{\bar{e}_{i}: 1 \leqslant i \leqslant s\right\}$ is a based manifold of rank $s$ and has $w$ as required. A concordance between such homeomorphisms $f, g$ gives a concordance of $g \circ f^{-1}$ to the identity. This extends the identity on the copies of $B_{s}^{ \pm}$to a based homeomorphism

$$
B_{s}^{ \pm} \cup_{f} B_{s}^{ \pm}=B_{s}^{ \pm} \cup_{1}\left(\partial B_{s}^{ \pm} \times I\right) \cup_{f} B_{s}^{ \pm} \rightarrow B_{s}^{ \pm} \cup_{1}\left(\partial B_{s}^{ \pm} \times I\right) \cup_{g} B_{s}^{ \pm}=B_{s}^{ \pm} \cup_{g} B_{s}^{ \pm} .
$$

Let $(M, \mathbf{e})$ be a based manifold of rank $s$. Then e determines a 3-connected map $B_{s}^{+} \rightarrow M$. As $\pi_{1}(0)=\pi_{1}(P L)=\pi_{1}(T O P)$ is detected by $w_{2}$, it follows from the immersion theorem $(4,2,9$,$) that this map is homotopic to an orientation-preserving immersion$ $B_{s}^{ \pm} \rightarrow M$ if $w=0, B_{s}^{-} \rightarrow M$ if $w \neq 0$. By general position we get an embedding. Embedding two disjoint copies of $B_{s}^{+}$in $M$ in this homotopy class (one orientationpreserving and one orientation-reversing), and using the $h$-cobordism theorem, we see that there is a homeomorphism of $\partial B_{s}^{ \pm}$of the type considered such that the corresponding based manifold is based homeomorphic to $(M, \mathbf{e})$. As

$$
\pi_{2}(0)=\pi_{2}(P L)=\pi_{2}(T O P)=0,
$$

a similar argument shows that the homeomorphism is unique up to concordance.

Concordance classes of orientation-preserving homeomorphisms of $\partial B_{s}^{ \pm}$which induce the identity on $H_{2}\left(\partial B_{s}^{ \pm}\right)$form a group under composition. Thus there are corresponding group structures on $\mathbf{M}_{s}^{+}$and $\mathbf{M}_{s}^{-}$.

Proposition 2. The cup-product, Pontrjagin class, and triangulation class define homomorphisms

$$
\begin{gathered}
\mu: \mathbf{M}_{s}^{ \pm} \rightarrow \operatorname{Hom}_{\mathbb{Z}}\left(\otimes^{3}\left(\oplus_{1}^{8} \mathbb{Z}\right), \mathbb{Z}\right), \\
p: \mathbf{M}_{s}^{ \pm} \rightarrow \operatorname{Hom}_{\mathbb{Z}}\left(\oplus_{1}^{8} \mathbb{Z}, \mathbb{Z}\right), \quad \Delta: \mathbf{M}_{s}^{ \pm} \rightarrow \operatorname{Hom}_{\mathbb{Z}}\left(\oplus_{1}^{s} \mathbb{Z}, \mathbb{Z}_{2}\right) .
\end{gathered}
$$

Proof. If $(M, \mathbf{e})$ represents an element of $\mathbf{M}_{s}^{ \pm}$, the base of $H^{2}(M)$ dual to e defines an isomorphism $\oplus_{1}^{s} \mathbb{Z} \rightarrow H^{2}(M)$. $\mu, p$ and $\Delta$ are then defined using the $\mu, p, \Delta$ of section 1 .

Now $\partial B_{s}^{ \pm} \simeq V_{1}^{s}\left(S^{2} \vee S^{3}\right) \cup e^{5}$. Let $\iota_{i} \in \pi_{2}\left(\partial B_{s}^{ \pm}\right), \iota_{i}^{\prime} \in \pi_{3}\left(\partial B_{s}^{ \pm}\right)$be the homotopy classes of the inclusion maps of the $i$ th copies of $S^{2}, S^{3}$. Let $f, g$ be homotopy classes of orientationpreserving homotopy equivalences of $\partial B_{s}^{ \pm}$inducing the identity on $H_{2}\left(\partial B_{s}^{ \pm}\right)$. By Poincaré duality, $f$ and $g$ induce the identity on $H_{3}\left(\partial B_{s}^{ \pm}\right)$. Then, by the Hilton-Milnor theorem (3), $f$ determines $\lambda_{j}^{i}(f), \lambda_{j k}^{i}(f) \in \mathbb{Z}, j>k$ such that

$$
f \circ \iota_{i}^{\prime}=\iota_{i}^{\prime}+\Sigma \iota_{j} \circ \eta \cdot \lambda_{j}^{i}(f)+\sum_{j>k}\left[\iota_{j}, \iota_{k}\right] \cdot \lambda_{j k}^{i}(f)
$$

where $\eta \in \pi_{3}\left(S^{2}\right)$ has Hopf invariant 1. As $f \circ \iota_{i}=\iota_{i}=g \circ \iota_{i}$, we have

$$
\lambda_{j}^{i}(g \circ f)=\lambda_{j}^{i}(g)+\lambda_{j}^{i}(f), \quad \lambda_{j k}^{i}(g \circ f)=\lambda_{j k}^{i}(g)+\lambda_{j k}^{i}(f) .
$$

Let $(M, \mathrm{e})$ be a based manifold corresponding to a concordance class of homeomorphisms with homotopy class $f \in\left[\partial B_{s}^{ \pm} ; \partial B_{s}^{ \pm}\right]$. Up to homotopy $M$ is obtained from $B_{8}^{ \pm} \simeq V_{1}^{s} S^{2}$ by attaching by the $f \circ \iota_{i}^{\prime}$ the 4-cells given by the base of $H_{4}(M)=H^{2}(M)$ 
dual to e, and then attaching a 6-cell. As $\eta$ has Hopf invariant 1, and the Whitehead product of a generator of $S^{2}$ with itself has functional product 1 , it follows as in ((13), thm. 4) that

$$
\begin{aligned}
\left\langle e_{i}^{\prime} \cup e_{j}^{\prime} \cup e_{k}^{\prime},[M]\right\rangle= & \lambda_{j}^{i}(f) \quad(j=k), \\
& \lambda_{j k}^{i}(f) \quad(j>k),
\end{aligned}
$$

where $\left\{e_{i}^{\prime}: 1 \leqslant i \leqslant s\right\}$ is the base of $H^{2}(M)$ dual to e, and $[M] \in H_{6}(M)$ is the fundamental class. Thus $\mu$ is a homomorphism.

Consider $M=B_{s}^{ \pm} \cup\left(\partial B_{s}^{ \pm} \times I\right) \cup B_{s}^{ \pm}$. As $B_{s}^{ \pm} \simeq V_{1}^{s} S^{2}$, there is a unique equivalence class of reductions to a $P L$ bundle of the restriction to $B_{s}^{ \pm} \cup B_{s}^{ \pm}$of the stable tangent bundle of $M$. The triangulation class of $M$ is the obstruction to extending this over $\partial B_{s}^{ \pm} \times I . \mathrm{As}$

$$
B_{s}^{ \pm} \cup_{\rho \circ f} B_{s}^{ \pm}=B_{s}^{ \pm} \cup_{g}\left(\partial B_{s}^{ \pm} \times I\right) \cup_{1}\left(\partial B_{s}^{ \pm} \times I\right) \cup{ }_{f} B_{s}^{ \pm},
$$

it follows that $\Delta$ is a homomorphism.

Similarly, the double of the stable tangent bundle has trivial restriction to $B_{s}^{ \pm} \cup B_{s}^{ \pm}$, and any two trivialisations are equivalent, for $w_{2}: \pi_{2}(B T O P) \rightarrow \mathbb{Z}_{2}$ is an isomorphism, and $\pi_{2}(T O P)=0$. The obstruction in $H^{4}\left(M ; \pi_{4}(B T O P)\right)$ to extending such a trivialisation over $\partial B_{s}^{ \pm} \times I$ maps to $2 p(M) \in H^{4}(M ; \mathbb{Z})$ under the change of coefficients given by the universal first Pontrjagin class (12). Thus $p$ is a homomorphism.

\section{Proposition 3.}

$$
\mu \oplus p \oplus \Delta: \mathbf{M}_{s}^{ \pm} \rightarrow \operatorname{Hom}_{\mathbb{Z}}\left(\otimes^{3}\left(\oplus_{1}^{s} \mathbb{Z}\right), \mathbb{Z}\right) \oplus \operatorname{Hom}_{\mathbb{Z}}\left(\oplus_{1}^{s} \mathbb{Z}, \mathbb{Z}\right) \oplus \operatorname{Hom}_{\mathbb{Z}}\left(\oplus_{1}^{s} \mathbb{Z}, \mathbb{Z}_{2}\right)
$$

is a monomorphism.

Proof. Let $(M, \mathbf{e})$ represent an element of $\mathbf{M}_{s}^{+}$in the kernal of $\mu \oplus p \oplus \Delta$. Then $M$ admits a $P L$ structure (8). By ((13), thm. 5) $(M, \mathrm{e})$ is based diffeomorphic to

with the standard base.

$$
\#{ }_{1}^{s} S^{2} \times S^{4}=B_{s}^{+} \cup_{1} B_{s}^{+}
$$

Now let $(M, \mathrm{e})$ represent an element of $\mathbf{M}_{s}^{-}$in the kernel of $\mu \oplus p \oplus \Delta$. We shall split $M$ as the connected sum of the non-trivial $S^{4}$-bundle over $S^{2}$ and $M_{0}$, where $M_{0}$ and the restriction of e represent an element of $\mathbf{M}_{s-1}^{+}$in the kernel of $\mu \oplus p \oplus \Delta$. Let $K$ be the complement in $M$ of a point. It follows from the proof of Proposition 2 that there is a homotopy equivalence $V_{1}^{s}\left(S^{2} \vee S^{4}\right) \rightarrow K$ taking chosen fundamental classes of the $i$ th $S^{2}, S^{4}$ to $e_{i} \in H_{2}(K)=H_{2}(M), e_{i}^{\prime} \in H_{4}(K)=H_{4}(M)=H^{2}(M)$, where $\left\{e_{i}^{\prime}: 1 \leqslant i \leqslant s\right\}$ is the base dual to e. Let $\iota_{i} \in \pi_{2}(K), \iota_{i}^{\prime} \in \pi_{4}(K)$ be the homotopy classes of the inclusions of the $i$ th $S^{2}, S^{4}$. An attaching map for the top cell of $M$ determines an element $\alpha \in \pi_{5}(K)$. By the Hilton-Milnor theorem(3),

$$
\begin{aligned}
\alpha=\Sigma \iota_{i} \circ \alpha_{1}+\Sigma \iota_{i}^{\prime} \circ \alpha_{i}^{\prime}+\sum_{i>j}\left[\iota_{i}, \iota_{j}\right] \circ \alpha_{i j} & +\sum\left[\iota_{i}, \iota_{j}^{\prime}\right] \circ \alpha_{i j}^{\prime} \\
& +\sum_{i>j \leqslant k}\left[\left[\iota_{i}, \iota_{j}\right], \iota_{k}\right] \circ \alpha_{i j k}+\Sigma\left[\left[\left[\iota_{i}, \iota_{j}\right], \iota_{k}\right], \iota_{1}\right] \circ \alpha_{i j k l},
\end{aligned}
$$

where $\alpha_{i} \in \pi_{5}\left(S^{2}\right) ; \alpha_{i j} \in \pi_{5}\left(S^{3}\right) ; \alpha_{i}^{\prime}, \alpha_{i j k} \in \pi_{5}\left(S^{4}\right) ; \alpha_{i j}^{\prime}, \alpha_{i j k l} \in \pi_{5}\left(S^{5}\right)$. (The Jacobi identity has been used to re-express Whitehead products of the form $\left[\left[\iota_{i}, \iota_{j}\right],\left[\iota_{k}, \iota_{1}\right]\right]$.) Considering intersection numbers we see that $\alpha_{i j}^{\prime}=\delta_{i j}$. The $\alpha_{i}^{\prime}$ determine $S q^{2}: H^{4}\left(M ; \mathbb{Z}_{2}\right) \rightarrow H^{6}\left(M ; \mathbb{Z}_{2}\right)$ which is dual to $w_{2}$, so $\alpha_{i}^{\prime}=0(i>1), \alpha_{1}^{\prime}=S^{2} \eta$. 
Suspension $\pi_{4}\left(S^{n}\right) \rightarrow \pi_{5}\left(S^{n+1}\right)$ is an isomorphism for $n=2,3,4$, and so is composition with $S^{2} \eta: \pi_{4}\left(S^{2}\right) \rightarrow \pi_{5}\left(S^{2}\right)$. Thus we can find $\beta_{j}^{i} \in \pi_{4}\left(S^{2}\right), \beta_{j k}^{i} \in \pi_{4}\left(S^{3}\right)(j>k)$,

such that

$$
\beta_{j k l}^{i} \in \pi_{4}\left(S^{4}\right) \quad(j>k \leqslant l)
$$

$$
\begin{gathered}
S \beta_{i j k}^{l}=-\alpha_{i j k l}, \\
S \beta_{j k}^{i}=-\delta_{k 1}\left(\alpha_{j 1 i}+\beta_{j 11}^{1} \circ S^{2} \eta+\delta_{i j}\left(1-\delta_{1 j}\right) \lambda\left(\beta_{j}^{1}\right)\right), \\
S \beta_{j}^{i}=-\delta_{i 1}\left(\alpha_{j 1}+\beta_{j 11}^{1} \circ S^{2} \eta\right) \quad(i, j) \neq(1,1), \\
\beta_{1}^{1} \circ S^{2} \eta=-\alpha_{1},
\end{gathered}
$$

where $\lambda: \pi_{4}\left(S^{2}\right) \rightarrow \pi_{5}\left(S^{4}\right)$ is $h_{2}$ of ((11), p. 37) followed by suspension. Then there is $f \in[K ; K]$ with $f \circ \iota_{i}=\iota_{i}$ and

$$
f \circ \iota_{i}^{\prime}=\Sigma \iota_{j} \circ \beta_{j}^{i}+\sum_{j>k}\left[\iota_{j}, \iota_{k}\right] \circ \beta_{j k}^{i}+\sum_{j>k \leqslant l}\left[\left[\iota_{j}, \iota_{k}\right], \iota_{1}\right] \circ \beta_{j k l}^{i} .
$$

By the Whitehead theorem, as $f$ induces isomorphisms in homology, it is a homotopy equivalence. Thus another homotopy class of an attaching map for the top cell of $M$ is

$$
\begin{aligned}
f \circ \alpha=\sum_{i>1} \iota_{i} \circ\left(\alpha_{i}+\beta_{i}^{1} \circ S^{2} \eta\right)+\iota_{1}^{\prime} \circ S^{2} \eta+\Sigma\left[\iota_{i}, \iota_{i}^{\prime}\right]+\sum_{i>j>1}\left[\iota_{i}, \iota_{j}\right] \circ \bar{\alpha}_{i j} \\
+\sum_{i>j>1, j \leqslant k}\left[\left[\iota_{i}, \iota_{j}\right], \iota_{k}\right] \circ \bar{\alpha}_{i j k},
\end{aligned}
$$

where $\bar{\alpha}_{i j} \in \pi_{5}\left(S^{3}\right), \bar{\alpha}_{i j k} \in \pi_{5}\left(S^{4}\right)$ (using Steer's theorem on Whitehead products of compositions ((11), 3.3)).

Put $C=S^{4} \vee\left(V_{1}^{s-1}\left(S^{2} \vee S^{4}\right)\right)$. Then there is a map of $C$ into $K$ taking the homotopy class of the $i$ th $S^{2}, S^{4}$ to $\iota_{i+1}, \iota_{i}^{\prime}$. Thus we can regard $\pi_{5}(C)$ as a subgroup of $\pi_{5}(K)$. Put $\gamma=f \circ \alpha-\iota_{1}^{\prime} \circ S^{2} \eta-\left[\iota_{1}, \iota_{1}^{\prime}\right]$. Then $\gamma \in \pi_{5}(C)$.

The total space of the non-trivial $S^{4}$-bundle over $S^{2}$ is the union of two copies of $D^{2} \times S^{4}$ with their boundaries identified by a map of $S^{1} \times S^{4}$ to itself. Composing this with projection onto $S^{4}$ and the inclusion in $C$ of the first copy of $S^{4}$, we get a map $g: S^{1} \times S^{4} \rightarrow C$. Then $C \cup_{g}\left(D^{2} \times S^{4}\right) \simeq K \cup_{c} e^{6}$, where $c=\iota_{1}^{\prime} \circ S^{2} \eta+\left[\iota_{1}, \iota_{1}^{\prime}\right] \quad((7), 3 \cdot 7)$. Altering $g$ on an embedded $D^{5}$ in $S^{1} \times S^{4}$ by a representative of $\gamma$ we get $h: S^{1} \times S^{4} \rightarrow C$ such that $C \cup_{h}\left(D^{2} \times S^{4}\right) \simeq K \cup_{f} o_{\alpha} e^{6} \simeq M$. Now the trivial $S^{1}$ - fibration over $S^{4}, O, g$, and such a homotopy equivalence define a Poincaré embedding of $S^{4}$ in $M$. (By ((14), $2 \cdot 7$ (ii)), $\left(C, S^{1} \times S^{4}\right)$ is a Poincaré pair.) As $(M, \mathrm{e})$ represents an element of ker $\Delta$, $M$ has a smooth structure. Now $\pi_{4}(G)=0$, so $\pi_{4}(G / O) \rightarrow \pi_{3}(O)$ is monomorphic. Also, the Pontrjagin class $p: \pi_{3}(O) \rightarrow \mathbb{Z}$ is monomorphic ((12), $\left.4 \cdot 1\right)$. As $(M, \mathrm{e})$ represents an element of $\operatorname{ker}(p)$, the tangential structure induced on $S^{4}$ by the Poincaré embedding is the natural one. By Browder's embedding theorem ((14), 11·3), there is a smooth embedding of $S^{4} \times D^{2}$ in $M$ inducing the above Poincaré embedding. Doing surgery on this we get a closed, oriented, smooth manifold $M_{0}$. Also, e determines a base $\mathbf{e}_{0}$ of $H_{2}\left(M_{0}\right)$. It can be seen that $\left(M_{0}, \mathbf{e}_{0}\right)$ represents an element of $\mathbf{M}_{s-1}^{+} \cap \operatorname{ker}(\mu \oplus p \oplus \Delta)$. Thus $\left(M_{0}, \mathrm{e}_{0}\right)$ is based diffeomorphic to \# $\#_{1}^{s-1} S^{2} \times S^{4}$ with the standard base. We recover $\left(M\right.$, e) by doing surgery on an embedded $S^{1} \times D^{5}$. Such embeddings are classified up to isotopy by an element of $\pi_{1}\left(O_{5}\right)=\mathbb{Z}_{2}$ determining the framing. As $M$ has $w \neq 0$, $(M, \mathrm{e})$ is based diffeomorphic to $B_{s}^{-} \cup_{1} B_{s}^{-}$with the standard base. 
Theorem 1. Orientation-preserving homeomorphism classes of closed, oriented, 1connected 6- manifolds with torsion-free homology correspond bijectively with isomorphism classes of systems of invariants:

$r$

$H$

$\mu: H \oplus H \oplus H \rightarrow \mathbb{Z}$

$w \in H \otimes \mathbb{Z}_{\mathbf{2}}$

$p \in \operatorname{Hom}_{\mathbb{Z}}(H, \mathbb{Z})$,

$\Delta \in \operatorname{Hom}\left(H, \mathbb{Z}_{2}\right)$,

satisfying

$$
\mu(2 x+W, 2 x+W, 2 x+W) \equiv(p+24 T)(2 x+W) \quad(\bmod 48)
$$

for all $x \in H$, where $W \in H, T=\operatorname{Hom}_{\mathbb{Z}}(H, \mathbb{Z})$ reduce $\bmod 2$ to $w, \Delta$. Such a manifold has a smooth (or $P L$ ) structure iff $\Delta=0$.

Proof. The invariants are defined in section 1. Uniqueness of manifolds with given invariants follows from Theorem 0 and Propositions 1, 2, 3, together with the remark that such a manifold $M$ with $H_{3}(M)=0$ always admits a base e making $(M, \mathbf{e})$ a based manifold.

Now let $r, H, \mu, w, p, \Delta$ be a system of invariants as above. We can choose a base $\left\{e_{i}: 1 \leqslant i \leqslant s\right\}$ of $H$ such that the dual base $\mathrm{e}^{\prime}=\left\{e_{i}^{\prime}: 1 \leqslant i \leqslant s\right\}$ satisfies $e_{i}^{\prime}(w)=0$ for $i>1$. We have $\partial B_{s}^{ \pm} \simeq V_{1}^{s}\left(S^{2} \vee S^{3}\right) \cup e^{5}$. Let $\iota_{i} \in \pi_{2}\left(\partial B_{s}^{ \pm}\right), \iota_{i}^{\prime} \in \pi_{3}\left(\partial B_{s}^{ \pm}\right)$be the homotopy classes of the $i$ th copies of $S^{2}, S^{3}$. Define $\lambda=\left(\lambda^{1}, \ldots, \lambda^{s}\right) \in \oplus_{1}^{8} \pi_{3}\left(\partial B_{s}^{ \pm}\right)$by

$$
\lambda^{i}=\iota_{i}^{\prime}+\Sigma \mu\left(e_{i}, e_{j}, e_{j}\right) \iota_{j} \circ \eta+\sum_{j>k} \mu\left(e_{i}, e_{j}, e_{k}\right)\left[\iota_{j}, \iota_{k}\right] \text {. }
$$

It follows from $((7), 3 \cdot 7)$ that the obstruction in $\pi_{4}\left(\partial B_{s}^{ \pm}\right)$to extending

$$
V_{1}^{s}\left(\iota_{i} \vee \lambda^{i}\right) \in\left[V_{1}^{s}\left(S^{2} \vee S^{3}\right) ; \partial B_{s}^{ \pm}\right]
$$

to an element of $\left[\partial B_{s}^{+} ; \partial B_{s}^{+}\right]$if $w=0,\left[\partial B_{s}^{-} ; \partial B_{s}^{-}\right]$if $w \neq 0$ is $\Sigma\left(\lambda^{i} \circ e_{i}^{\prime}(w) S \eta+\left[\iota_{i}, \lambda^{i}\right]\right)$. Using symmetry of $\mu$, the given congruence relation, Steer's theorem ((11), 3.3), and the Jacobi identity, we see that this vanishes. Thus there is a map $f$ of $\partial B_{s}^{ \pm}$to itself as required. $f$ induces isomorphisms in homology, so is a homotopy equivalence. $B_{s}^{ \pm} \cup_{f} B_{s}^{ \pm}$ is therefore a Poincaré complex, and by ((13), thm. 8) it has the homotopy type of a smooth manifold $M$. If $\bar{p} \in H^{4}(M ; \mathbb{Z})$ is the first Pontrjagin class of $M, M$ has invariants $0, H, \mu, w, \bar{p}, 0$. Then for $x \in H, \bar{p}(x) \equiv p(x)(\bmod 24)$, and if $w\left(e_{1}\right) \neq 0$,

$$
\bar{p}\left(e_{1}\right) \equiv(p+24 T)\left(e_{1}\right) \quad(\bmod 48) \text {. }
$$

By ((13), thm. 5) there is a smooth based manifold $\left(M_{0}, \mathrm{e}_{0}\right)$ of rank 1 with vanishing cup-product and Stiefel-Whitney class, and with Pontrjagin class 24 . It follows from Propositions 1 and 2 that, by adding to the class of $\left(M, \mathrm{e}^{\prime}\right)$ in $\mathbf{M}_{s}^{ \pm}$the class of suitable connected sums of $\left(M_{0}, \mathrm{e}_{0}\right)$ with copies of $S^{4}$ bundles over $S^{2}$ with standard bases, we can obtain the class of a based manifold $\left(M_{1}, \mathbf{e}_{1}\right)$ with invariants $0, H, \mu, w, p_{1}, 0$ where $p_{1} \equiv p+24 T(\bmod 48) \cdot\left(p-p_{1}\right) / 24$ defines an element of

$$
H^{4}\left(M_{1} ; \mathbb{Z}\right)=H^{4}\left(M_{1} ; \pi_{4}(G / T O P)\right) \text {. }
$$

This determines the homotopy class of a map of a 4-skeleton of $M_{1}$ into G/TOP which is trivial on a 2 -skeleton. As $\pi_{5}(G / T O P)=0$, this extends to an element of $\left[M_{1} ; G / T O P\right]$. 
Altering the normal bundle of $M_{1}$ by this gives a lift to a stable topological bundle $\xi$ of the stable normal spherical fibration of $M_{1}$. As the map given by the Pontrjagin class $\pi_{4}(B O) \rightarrow \mathbb{Z}$ is multiplication by $2((\mathbf{1 2}), 4 \cdot 1)$, and using $((\mathbf{1 0}), 5 \cdot 5), \xi$ has Pontrjagin class $p$ and triangulation class $\Delta$.

The above reduction of the stable normal spherical fibration of $M_{1}$ to $\xi$ gives rise to a surgery problem. There is an obstruction in $\pi_{6}(G / T O P)$ to doing the surgery. Altering the reduction over the top cell of $M_{1}$ by the obstruction we obtain a reduction to a stable topological bundle $\xi_{1}$ with surgery obstruction zero. Thus we may do surgery to get a manifold $M_{2}$ homotopy equivalent to $M_{1}$ with stable normal bundle $\xi_{1}$. As $\xi, \xi_{1}$ are isomorphic over a 4-skeleton, $M_{2}$ has Pontrjagin class $p$ and triangulation class $\Delta$. Finally $M_{2} \#\left(\#_{1}^{r} S^{3} \times S^{3}\right)$ has invariants $r, H, \mu, w, p, \Delta$ as required.

The final statement follows from $((10), \S 5)$.

CoRollary. Two closed, 1-connected, smooth 6-manifolds with torsion-free homology are diffeomorphic iff they are (topologically) homeomorphic.

There is also a homotopy classification.

Theorem 2. Orientation-preserving homotopy-equivalence classes of oriented, 1connected, finite Poincaré complexes of dimension 6 with torsion-free homology and first exotic class zero correspond bijectively with isomorphism classes of systems of invariants:

$r$

$H$

$\mu: H \otimes H \otimes H \rightarrow \mathbb{Z}$

$w \in H \otimes \mathbb{Z}_{2}$,

$h \in \operatorname{Hom}_{\mathbb{Z}}\left(H, \mathbb{Z}_{48}\right)$,

satisfying

$\rho_{48} \mu(2 x+W, 2 x+W, 2 x+W)=h(2 x+W)$

for all $x \in H$ where $W \in H$ reduces $\bmod 2$ to $w$ and $\rho_{48}$ is reduction $\bmod 48$.

Each such Poincaré complex has the homotopy type of a smooth manifold.

Proof. As the first exotic class $\pi_{2}(G / O) \rightarrow \mathbb{Z}_{2}$ is an isomorphism, the final statement follows from the proof of ((13), thm. 8).

Given such a Poincaré complex, $r, H, \mu, w$ are defined as the corresponding invariants of a smooth manifold of the same oriented homotopy type, and are clearly oriented homotopy type invariants. $h$ is the reduction mod 48 of the Pontrjagin class of any smooth manifold of the given oriented homotopy type. By the argument at the end of section $1, h$ is well defined. The relation follows from that of Theorem 1.

Given two such Poincaré complexes with isomorphic systems of invariants, represent them by oriented smooth manifolds $M_{1}, M_{2}$. The Pontrjagin classes of $M_{1}$ and $M_{2}$ are congruent mod 48. By the argument at the end of the proof of Theorem 1, we can alter the lift to a vector bundle of the stable normal spherical fibration of $M_{2}$ and do surgery to get an oriented smooth manifold $M_{3}$, oriented homotopy equivalent to $M_{2}$ and with the same Pontrjagin class as $M_{1}$. By Theorem 1, $M_{1}$ and $M_{3}$ are oriented homeomorphic, and so $M_{1}$ and $M_{2}$ are oriented homotopy equivalent. 


\section{REFERENCES}

(1) Bonrdman, J. M. and Vogt, R. M. Homotopy-everything $H$-spaces. Bull. Amer. Math. Soc. 74 (1968), 1117-1122.

(2) Haefliger, A. and Poenaro, V. La classification des immersions combinatoires. Publ. Math. I.H.E.S. 23 (1964), 75-91.

(3) Hilton, P. J. On the homotopy groups of the union of spheres. J. London Math. Soc. 30 (1955), 154-172.

(4) HIRSCH, M. W. Immersions of manifolds. Trans. Amer. Math. Soc. 93 (1959), 242-276.

(5) HIRZeBruch, F. Topological methods in algebraic geometry, 3rd ed. (Springer.Verlag, Berlin, 1966.)

(6) Hosemolter, D. Fibre bundles (McGraw-Hill, New York, 1966).

(7) JAMEs, I. M. and Whitehead, J.H. C. The homotopy theory of sphere bundles over spheres (I). Proc. London Math. Soc. (3), 4 (1954), 196-218.

(8) KTrby, R. C. and StebenmanN, L. C. On the triangulation of manifolds and the Hauptvermutung. Bull. Amer. Math. Soc. 75 (1969), 742-749.

(9) LEES, J. A. Immersions and surgeries of topological manifolds. Bull. Amer. Math. Soc. 75 (1969), 529-534.

(10) Stebenmann, L. C. A report on topological manifolds. Proceedings I.C.M. Nice (1970).

(11) SteEr, B. Generalized Whitehead products. Quart. J. Math. Oxford Ser. (2), 14 (1963), $29-40$.

(12) Tamora, I. On Pontrjagin classes and homotopy types of manifolds. J. Math. Soc. Japan 9 (1957), 250-262.

(13) WALL, C. T. C. Classification problems in differential topology. V. On certain 6-manifolds. Invent. Math. 1 (1966), 335-374.

(14) WALL, C. T. C. Surgery on compact manifolds (Academic Press, London, 1970). 